INPLASY

PROTOCOL

To cite: Cui et al. Traditional Chinese Medicine ultrasonic atomization influence on dry eye disease: A protocol for systematic review and metaanalysis. Inplasy protocol

202180061. doi:

10.37766/inplasy2021.8.0061

Received: 15 August 2021

Published: 15 August 2021

Corresponding author:

Yanru Cui

429731354@qq.com

Author Affiliation:

Shandong University of

Traditional Chinese Medicine.

Support: 2019XZZX-YK009.

Review Stage at time of this submission: The review has not yet started.

Conflicts of interest:

None declared.

\section{Traditional Chinese Medicine ultrasonic atomization influence on dry eye disease: A protocol for systematic review and meta-analysis}

Cui, Y1; Wang, J2; Wang, G3; Xie, X4; Tian, L5; Guo, C6.

Review question / Objective: Traditional Chinese medicine ultrasonic atomization therapy has become a novel focus niche in dry eye research and treatment as it can maintain constant temperature, humidity and drug concentration without the use of preservatives, reduce ocular surface toxicity of eye drops and, additionally, avoid hepatic first-pass effect. However, the clinical evidence in support of such pharmaceutical benefits is still presently limited. Consequently, this systematic review and meta-analyses aim to comprehensively explore and compile the effects of traditional Chinese medicine ultrasonic atomization on dry eye disease, in an effort to provide a more robust foundation for the clinical utilization of this technique that is based upon high-quality experimental evidence.

INPLASY registration number: This protocol was registered with the International Platform of Registered Systematic Review and Meta-Analysis Protocols (INPLASY) on 15 August 2021 and was last updated on 15 August 2021 (registration number INPLASY202180061).

\section{INTRODUCTION}

Review question / Objective: Traditional Chinese medicine ultrasonic atomization therapy has become a novel focus niche in dry eye research and treatment as it can maintain constant temperature, humidity and drug concentration without the use of preservatives, reduce ocular surface toxicity of eye drops and, additionally, avoid hepatic first-pass effect. However, the clinical evidence in support of such pharmaceutical benefits is still presently limited. Consequently, this systematic review and meta-analyses aim to comprehensively explore and compile the effects of traditional Chinese medicine ultrasonic atomization on dry eye disease, 
in an effort to provide a more robust foundation for the clinical utilization of this technique that is based upon high-quality experimental evidence.

Condition being studied: Dry eye (DE), is a chronic and progressive ophthalmic condition that leads to surface lesions, can cause burning, dryness sensation, pain, itching, heaviness and fatigue in the eyes, together with blurred vision and other symptoms. Its hallmark pathology is unstable tear film development in the eyes. Recent epidemiological studies have shown that $D E$ is a lifestyle-influenced disease. With the rapid development of cellphones, computer-based work and home entertainment in recent years, individual lifestyles and working environments have changed rapidly. The incidence of DE disease is increasing on a daily basis, consequently contributing to a reduced quality of life, affecting a large proportion of the global population. Severe DE cases exhibit symptoms ranging from limited daily activities to pain and reduced general health status, according to recent findings.

\section{METHODS}

Participant or population: Patients diagnosed with dry eye or keratoconjunctivitis sicca will be included in this analysis, with no restrictions as a function of patient age, sex, ethnicity, or educational status.

Intervention: Patients in the experimental group will be those that underwent both TCM ultrasonic atomization and routine treatment.

Comparator: Control patients will be individuals that underwent standard medical treatment.

Study designs to be included: All relevant randomized controlled trials (RCTs) will be eligible for inclusion.

Eligibility criteria: All relevant randomized controlled trials (RCTs) will be eligible for inclusion. Patients diagnosed with dry eye or keratoconjunctivitis sicca will be included in this analysis, with no restrictions as a function of patient age, sex, ethnicity, or educational status. Control patients will be individuals that underwent standard medical treatment, while patients in the experimental group will be those that underwent both TCM ultrasonic atomization and routine treatment. Primary findings will include tear secretion volume and Break-up time.

Information sources: We will search the PubMed, MEDLINE, Embase, Cochrane Clinical Trials Database, China National Knowledge Infrastructure, Wanfang Database, Chinese Science Journal Database, and China Biology Medicine databases for all studies published as of July 2021. Table 1 details the PubMed search strategy that will be employed for this study, and an identical strategy will be used for all other databases.

Main outcome(s): Primary findings will include tear secretion volume and Breakup time.

Additional outcome(s): 1.Ocular Surface Disease Index; 2.Corneal fluorescein staining; 3.Tear osmolarity.

Quality assessment / Risk of bias analysis: Risk of bias for the included RCTs will be assessed using the Cochrane Handbook Version 5.1.0 tool based on the following criteria, each of which will be noted as exhibiting a high, low, or uncertain risk of bias: randomization approach, assignment concealment, subject blinding methodology, result evaluation blinding methodology, selective reporting, data integrity, and other biases. Disagreements between reviewers will be resolved through discussion and, if required, through discussion with the third investigator.

Strategy of data synthesis: Statistical analyses will be conducted using RevMan 5.3.0. The 12 statistic and Chi-squared tests will be employed to detect heterogeneity among included RCTs, with random-effects models being used for pooled analyses in the presence of high heterogeneity 
$(12>50 \%)$, and fixed-effects models otherwise being used. Odds ratios (ORs) and $95 \%$ confidence intervals (Cls) will be employed to analyze dichotomous variables, whereas continuous variables will be evaluated using mean difference (MD) values and $95 \%$ Cls.

Subgroup analysis: When sufficient data are available or significant heterogeneity is detected, subgroup analyses will be performed based upon different interventions, controls, and outcome measures in particular trials.

Sensitivity analysis: Sensitivity analyses will be performed to confirm the stability of outcome indices.

Country(ies) involved: China.

Keywords: ultrasonic atomization, metaanalysis, protocol, dry eye.

Contributions of each author:

Author 1 - Yanru Cui.

Author 2 - Jilin Wang.

Author 3 - Gaofeng Wang.

Author 4 - Xiuguo Xie.

Author 5 - Lizhen Tian.

Author 6 - Chengwei Guo. 